\title{
Immunoglobulin G4 antibodies to rat urinary allergens, sensitization and symptomatic allergy in laboratory animal workers
}

\author{
L. Portengen, G. de Meer, G. Doekes and D. Heederik \\ Institute for Risk Assessment Sciences (IRAS), Utrecht University, The Netherlands
}

\begin{abstract}
Summary
Background and objectives We have previously reported that high rat urinary allergen (RUA) exposure was not associated with increased risk of rat allergy in long-term-exposed laboratory animal (LA) workers. We aimed to assess whether strong allergen-specific IgG4 responses could explain the absence of a dose response in these subjects. We investigated whether IgG4 was associated with allergen exposure and prevalence of sensitization or respiratory symptoms to rats. The longitudinal relation between $\mathrm{IgG} 4$ and rat allergy was studied using data obtained during 2 years of follow-up.

Methods Five hundred and twenty-nine LA workers answered a questionnaire on respiratory symptoms and occupational history and participated in skin prick testing. Blood samples were analysed for specific IgG4 and IgE to RUA. Exposure to RUA was estimated based on personal air samples. The relation between IgG4 and newly occurring sensitization or rat allergy was studied in workers who were not sensitized or did not report respiratory symptoms to rats.

Results IgG4 titres were higher in atopic than in non-atopic subjects, and increased with higher allergen exposure. Titres were highest in subjects who were sensitized and reported respiratory symptoms to rats when compared with those who were not (geometric mean [geometric standard deviation] $=202$ [5.7] vs. 8.4 [18.3] AU). The association between IgG4 and sensitization or symptomatic rat allergy was independent of estimated allergen exposure. IgG4 was a strong predictor of newly occurring sensitization and symptomatic rat allergy during follow-up in atopic and rat-sensitized subjects.

Conclusion High exposure to RUA is associated with a strong allergen-specific IgG4 antibody response. High anti-RUA IgG4 is a strong predictor of prevalent and incident sensitization and symptomatic rat allergy in atopic and rat-sensitized subjects. IgG4 can therefore not explain the absence of a dose response between allergen exposure and allergy in long-term-exposed workers. We consider anti-RUA IgG4 to be a marker that combines aspects of exposure and susceptibility.
\end{abstract}

Keywords allergy and immunology, follow-up studies, IgG4, laboratory animals, occupational disease, occupational exposure

Submitted 23 September 2003; revised 8 April 2004; accepted 11 May 2004

\section{Introduction}

Several studies have investigated exposure response relationships for sensitization to common and occupational allergens in domestic and specific occupational environments (as reviewed by [1-3]). Sensitization occurs for different allergens at very different exposure levels indicating that considerable differences exist in sensitizing potential [3]. Few studies have evaluated the shape of the exposure response relationships for allergic sensitization in detail, but there is evidence from cross-sectional studies that for some allergens sensitization risk levels off at higher exposure levels or is even bell shaped [4, 5].

In a study among children aged 12-14 years, highest exposure to the major cat allergen Fel d 1 was associated with

Correspondence: Lützen Portengen, Institute for Risk Assessment Sciences (IRAS), Utrecht University, PO Box 80176, 3508 TD, Utrecht, The Netherlands. E-mail: L.Portengen@iras.uu.nl a lower prevalence of sensitization (as determined by RAST) [5]. High exposure to cat allergen was associated with an IgG antibody response in the absence of sensitization and with a low risk of asthma. IgG antibodies to Fel d 1 included a large proportion of $\mathrm{IgG4}$, an isotype of which the production is dependent on IL-4, and the authors therefore referred to this as a modified T-helper type $2(\mathrm{Th} 2)$ response. As those with the $\operatorname{IgG}(4)$ response were also not symptomatic, this was considered a form of high-dose tolerance. Others have suggested that bell-shaped exposure response relationships may be the result of allergen exposure avoidance by subjects at high risk for allergies [4, 6].

From studies on occupational allergy it has long been known that the presence of detectable levels of allergenspecific IgG4 is more common in those with prolonged exposure to high doses of the allergen involved [7-9]. In these studies, presence of IgG4 was not associated with symptomatic allergy, and was therefore regarded as a marker of 
exposure. Studies on desensitization immunotherapy have shown that successful therapy is associated with the induction of high levels of allergen-specific IgG4, although this is now thought to reflect the induction of regulatory $\mathrm{T}$ cells, rather than being a causal factor in the outcome [10].

We have previously reported that in laboratory animal (LA) workers a clear dose-response relation between allergen exposure and risk of sensitization and symptomatic rat allergy could only be found in workers who had been working with LAs for a relatively short period [11]. As sensitization and symptoms to rat allergens were less prevalent at high exposure levels among workers with long job histories, the absence of a dose response in those workers was attributed to healthy worker selection. However, alternative explanations, e.g. a form of high-dose tolerance in at least some of these workers, could not be ruled out.

In this paper, we investigate whether the presence of high levels of allergen-specific IgG4 could be responsible for the absence of a dose response in workers with longer job histories in this cohort. We studied the relation between exposure to rat urinary allergens (RUAs) and $\mathrm{IgG} 4$ response and compared $\mathrm{IgG} 4$ levels in allergic and non-allergic workers. We also investigated whether levels of IgG4 predict development of sensitization or allergic respiratory symptoms to rat allergens during follow-up.

\section{Materials and methods}

\section{Study design and subjects}

Data were from a cross-sectional survey conducted in 1992/ 1993, and repeated in 1993/1994 and 1994/1995. Detailed description of the design and methods of data collection have been reported previously [11]. Each survey included a questionnaire and skin prick testing. A total of 586 workers participated in the baseline study and IgG4 to RUA was assessed in sera from $529(90 \%)$. Four hundred and fifty three and 381 subjects were seen again during the first and second follow-up, respectively, 466 workers $(88 \%)$ were seen at least once during follow-up. Sixty-three workers were lost to follow-up either because they did not work anymore in the LA facility or because they had holidays or were ill during the field study periods.

Seventy-seven $(15 \%)$ reported respiratory symptoms (chest tightness, sneezing, or a runny nose) attributed to working with rats at the first survey, and of those without symptoms $399(88 \%)$ were seen at least once during follow-up. Valid skin prick test (SPT) data from the first survey were available for 470 workers, and $86(18 \%)$ had a positive SPT to rat urine or rat hair. Of those with a negative SPT result, $315(82 \%)$ had valid SPTs from at least one follow-up survey. Follow-up cohorts for incident symptomatic allergy and sensitization therefore consisted of 399 and 315 subjects, respectively.

\section{Questionnaire}

The questionnaire contained questions on respiratory symptoms, airway hyper-responsiveness, allergic symptoms due to common and occupational allergens, smoking history, contact with LAs, and occupational history [12]. For his paper, the following questionnaire items were used.
Chronic asthma was defined as the presence of symptoms of shortness of breath, wheeze (ever), or chest tightness, while chronic bronchitis was defined as the presence of symptoms of cough or phlegm. Presence of symptoms of allergy to common allergens was defined on the basis of self-reported allergic symptoms (chest tightness, runny or sneezing nose, runny or itchy eyes, and itchy or red skin) to house dust, pollen, and/or domestic animals. Presence of symptoms of hyper-responsiveness was defined on the basis of self-reported difficulties in breathing when going from warm to cold surroundings, from cold to warm surroundings, during foggy weather and/or during cold weather. Symptomatic respiratory rat allergy was defined as the presence of self-reported respiratory symptoms (chest tightness, sneezing, or a runny nose) attributed to working with rats.

\section{Exposure assessment}

Individual work histories were recorded at each visit and subjects were asked how long they had been working with LAs prior to enrolment in the study. Detailed information on the quantification of RUAs exposure can be found elsewhere [13]. Based on the information collected during the visits, intensity of exposure (in three categories) was estimated for each subject [11]. Subjects were asked to estimate the number of hours they had worked with rats, feces, or urine during an appropriate interval in the year before the visit. Exposure to RUA was defined as the product of exposure intensity and the number of hours a subject had worked with rats or rat products during the year preceding the survey, as earlier cross-sectional analyses in this population had revealed that this predicted specific skin test sensitization best.

Based on the number of years a subject had been working with LAs at the first survey, exposure duration was classified as either short term ( $\leqslant 4$ years) or long term ( $>4$ years) in keeping with earlier reports on this population [11].

\section{Skin prick testing}

Skin prick testing was performed as described previously [14] with five common allergens (mixture of Dermatophagoides pteronyssinus and $D$. farinae, mixture of grass pollen, mixture of tree pollen, cat fur, dog fur, all from ALK Benelux, Houten, the Netherlands), six occupational allergens (rat urine; rat fur; mouse urine; mouse fur; rabbit fur; guinea-pig fur, all from ALK Benelux) and positive (histamine $10 \mathrm{mg}$ / $\mathrm{mL}$ ) and negative (phosphate-buffered saline, PBS) controls. Common atopy was defined as a positive SPT (weal size $\geqslant 3 \mathrm{~mm}$ ) to at least one common allergen. Sensitization to rats was defined as a positive SPT to rat urine or rat fur.

\section{IgG4 serology}

Sera were stored at $-20^{\circ} \mathrm{C}$ until analysis. Specific $\mathrm{IgG} 4$ antibodies to RUA were determined by a modified EIA as described previously [8]. Microwells were coated overnight at $4{ }^{\circ} \mathrm{C}$ with $200 \mu \mathrm{L}$ of a $10 \mu \mathrm{g} / \mathrm{mL}$ preparation of rat urinary proteins in PBS $(\mathrm{pH}=7.0)$. Details of the allergen preparation have been described by Hollander et al. [13]. After blocking with PBS containing $0.05 \% \mathrm{v} / \mathrm{v}$ Tween-20 and $0.2 \%$ $\mathrm{w} / \mathrm{v}$ gelatine $(\mathrm{PBTG} ; \mathrm{pH}=7.4)$, sera were tested at $1 / 2$ in PBTG in duplicate. After incubation for $1 \mathrm{~h}$ at $37^{\circ} \mathrm{C}$ binding 
of specific IgG4 was determined by incubation with peroxidase-labelled monoclonal mouse anti-human IgG4 (CLB, Amsterdam, the Netherlands) diluted $1: 1000$ in PBTG $\left(1 \mathrm{~h}\right.$ at $37^{\circ} \mathrm{C}$ ), followed by incubation for $30 \mathrm{~min}$ at $20^{\circ} \mathrm{C}$ with $2 \mathrm{mg} / \mathrm{mL}$ OPD (Sigma, St Louis, MO, USA) in citrate/ phosphate buffer $(\mathrm{pH}=5.5)$, containing $0.015 \% \mathrm{v} / \mathrm{v}$ hydrogen peroxide. The reaction was stopped by adding $50 \mu \mathrm{L}$ of a $2 \mathrm{M} \mathrm{HCl}$ solution and the optical density (OD) read on a spectrophotometer at a wavelength of $405 \mathrm{~nm}$. Antibody concentrations were expressed as relative titres, calculated by interpolation of the logit-transformed OD values on a calibration line obtained in the same microtitre plate with a pool of strongly positive sera, which was arbitrarily given an antibody titre of 100 . For samples with a logit value above +3 , the analysis was repeated at higher dilutions (10-2000).

\section{IgE serology}

Specific IgE antibodies to RUA were measured by immunoassay (AlaSTAT; DPC, Apeldoorn, the Netherlands) [15].

\section{Statistical analysis}

Crude differences in prevalence rates between groups were compared using Fisher's exact test, while $F$-tests were used to compare the means (or geometrical means) of continuous variables. Simple bivariate relations between two continuous variables were investigated by correlation analysis, using Spearman's rank correlation coefficient $(\rho)$ whenever applicable.

Exposure to RUA and titres of IgE and IgG4 to RUA did not follow a normal distribution, and was log transformed and presented as geometric mean (GM) and geometric standard deviation (GSD) where necessary. For subjects not working with rats, exposure was arbitrarily set at two-thirds of the lowest calculated exposure level in those working with rats $\left(1.18 \mathrm{ng} / \mathrm{m}^{3} \times \mathrm{h} / \mathrm{yr}\right)$ before $\log$ transformation. Titres of IgG4 below the detection limit were replaced by two-thirds of the limit of detection (0.001 AU) before log transformation. All statistical analyses were performed using SAS software (version 8.02; SAS Institute, Cary, NC, USA).

Exposure response relations for exposure to RUA and levels of IgG4 and for IgG4 levels and sensitization or symptomatic allergy were explored by non-parametric regression modelling (smoothing) using PROC GAM. Atopy and a positive SPT to rat allergens were effect modifiers for the relation between $\mathrm{IgG} 4$ and sensitization or symptomatic rat allergy, respectively, and were therefore used to define strata for stratified analyses.

For dichotomous response variables, a logistic model was used, and smoothed curves were computed using a logit-link function and transformed to prevalence or cumulative incidence by applying the inverse of the logit function. The smoothness of the function was initially determined by generalized cross-validation ( $\operatorname{method}=\mathrm{GCV}$ ), but the number of degrees of freedom was not allowed to exceed a maximum of 3 to avoid extremely irregular curves. Model selection was done informally by comparing deviances and favouring the simplest model when there was little difference between two models.

All statistical tests were done two-sided and a $P$-value $<0.05$ was considered significant.

\section{Results}

\section{Subject characteristics}

Subject characteristics for the study population available in the cross-sectional analysis are presented in Table 1. Three hundred and twenty-seven subjects $(62 \%)$ had been working with LAs for more than 4 years (long-term-exposed workers). Prevalences of atopy, rat sensitization, and symptomatic rat allergy were similar for short-term and long-term-exposed workers, but exposure to RUA was significantly higher in the latter group.

\section{Prevalent rat allergy and exposure to RUA}

Using non-parametric models to describe the relation between allergen exposure and sensitization, the strong association between exposure and the prevalence of a positive SPT to rat allergens and symptomatic rat allergy in atopic shortterm-exposed workers was confirmed (Fig. 1). For atopic long-term-exposed workers risk of sensitization and symptomatic allergy did not increase with exposure to RUA and even declined at the highest exposure levels. Prevalence of a positive SPT and symptomatic allergy were low in non-atopic and non-sensitized workers, respectively.

\section{IgG4 antibody titres and exposure to RUA}

IgG4 antibodies to RUA could be detected in sera from 439 of 529 subjects $(83 \%)$. Log-transformed titres followed an approximate normal distribution with a GM [GSD] of 14.6 [18.4] AU. In non-parametric regression analysis, a clear dose response with exposure to RUA was found for both atopic and non-atopic workers. Atopic workers had higher levels of IgG4 than non-atopic workers, but the shape of the doseresponse curve was similar for both groups (Fig. 2). IgG4 levels weakly increased with job duration for subjects who

Table 1. Descriptive statistics of selected subject characteristics

\begin{tabular}{lc}
\hline & $n(\%) /$ mean (SD) \\
\cline { 2 - 2 }$N$ & 529 \\
\hline Gender (female) & $206(39 \%)$ \\
Smoker* & $133(25 \%)$ \\
Former smoker* & $145(27 \%)$ \\
Age & $35.3(9.6)$ \\
Chronic respiratory symptoms & \\
$\quad$ Asthma & $137(26 \%)$ \\
$\quad$ Bronchitis & $46(9 \%)$ \\
Symptoms of allergy to common allergens & $146(28 \%)$ \\
Symptoms of hyper-responsiveness & $78(15 \%)$ \\
Previous working experience with LAs (years) & $10.4(9.7)$ \\
Yearly hours working with rats (h/yr) $\dagger$ & $516(534)$ \\
Cumulative exposure to rat allergen during previous year & $152[5.1]$ \\
$\quad$ (ng/m ${ }^{3} \times$ h/yr) GM [GSD] $\dagger$ & \\
Atopic $\ddagger$ & $210(45 \%)$ \\
Sensitized to rats (SPT) $\ddagger$ & $86(18 \%)$ \\
Respiratory symptoms of rat-allergy & $77(15 \%)$ \\
\hline
\end{tabular}

*Information on smoking habits was missing for one subject. $\dagger$ In 373 subjects reporting to work with rats. Information on exposure to rats was missing for one subject. ‡SPT were available for 470 subjects.

SPT, skin prick test; GM, geometric mean; GSD, geometric standard deviation; SD, standard deviation; LA, laboratory animal. 

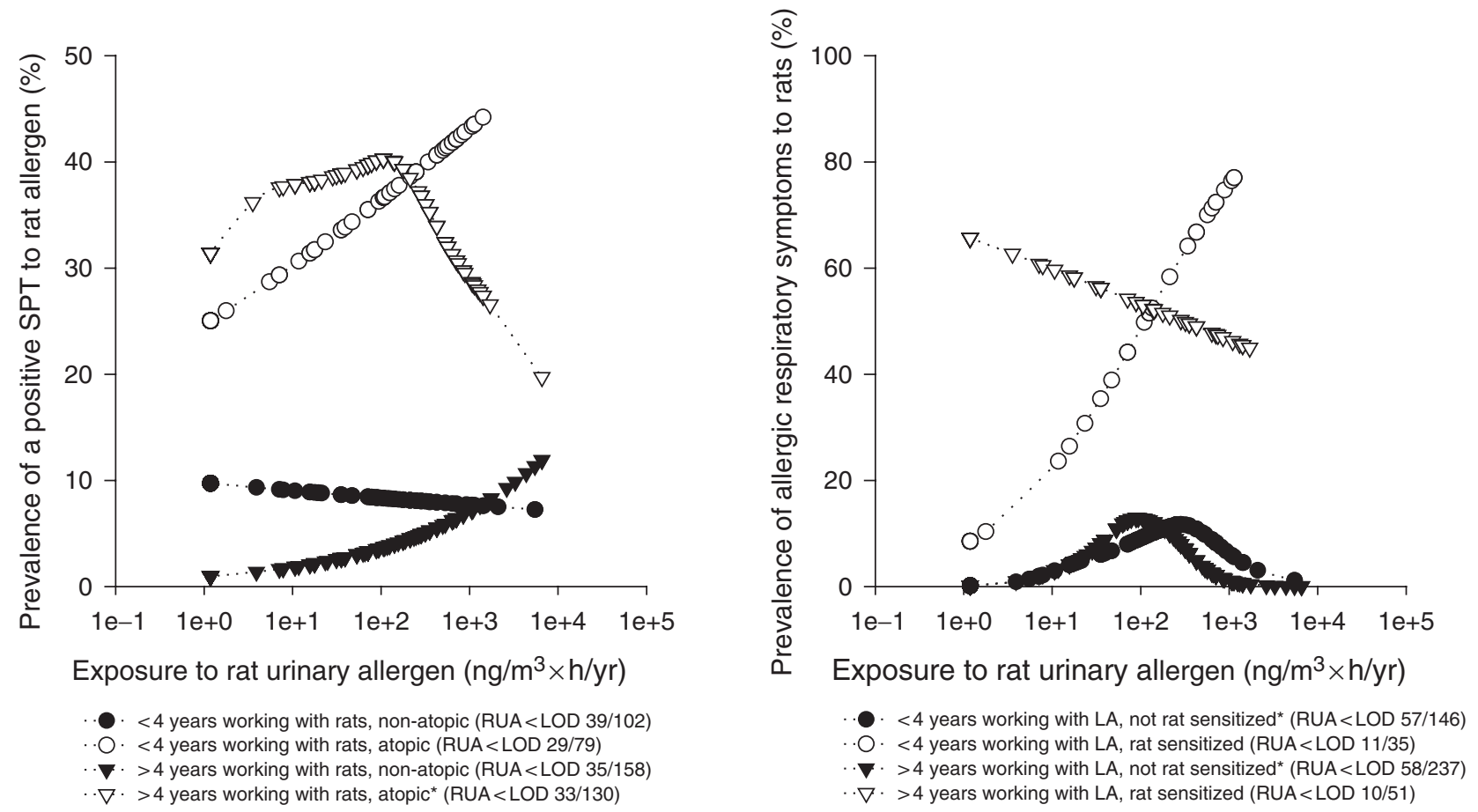

Fig. 1. Smoothed (cubic spline) plots of exposure to rat urinary allergen (RUA) and prevalent sensitization (skin prick test, SPT) or symptomatic allergy to rats in long-term and short-term- exposed laboratory animal (LA) workers. Smoothness of the plot determined by generalized cross-validation except where indicated in the legend with a * (see Materials and methods section). Subjects not working with rats during the year preceding the study were assigned an arbitrary exposure of $1.18 \mathrm{ng} / \mathrm{m}^{3} \times \mathrm{h} / \mathrm{yr}$ before log transformation (see Materials and methods section). The number of subjects not working with rats is indicated in the legend as: RUA < limit of detection (LOD) number of subjects/total number of subjects in group. For higher exposures a single dot represents one to 11 subjects (median $=1$ ), depending on the number of subjects with identical estimated exposure in a particular group.

had been working with LAs for less than 10 years, but showed no consistent trend for those with more extensive job histories (not shown).

IgG4 antibody levels and prevalent rat allergy

In a first attempt to assess whether IgG4 was associated with the lack of a dose response in long-term-exposed workers, we compared IgG4 titres in subjects grouped according to the presence or absence of a positive SPT and/or respiratory symptoms to rat allergens (Fig. 3). Titres of $\mathrm{IgG} 4$ in longterm-exposed workers were highest in those who were sensitized and reported respiratory symptoms $(\mathrm{GM}[\mathrm{GSD}]=$ 202 [5.7] vs. 8.4 [18.3] in non-sensitized, asymptomatic subjects), but were also slightly higher in sensitized subjects who did not report symptoms (GM [GSD] = 30 [17.9]). Titres were similar for long-term- and short-term-exposed workers, both within subgroups and overall.

Both weal diameter and serum titre of anti-RUA $\operatorname{IgE}$ correlated with IgG4 titres in workers with a positive SPT to rat allergens, although the correlation was not very strong (Spearman's $\rho=0.34$ and 0.32 , respectively; $P<0.01$ ).

To evaluate whether an inverse association between IgG4 response and rat allergy could exist at very high or very low exposure levels, we further analysed the association between IgG4 and sensitization or symptomatic rat allergy in longterm-exposed workers exposed at three different exposure levels (Fig. 4). Sensitization risk strongly increased with increasing IgG4 at all exposure levels, and only appeared to level off or decrease at titres above $100 \mathrm{AU}$ in subjects who did not work with rats. The association between IgG4 and prevalence of symptomatic rat allergy within different exposure categories appeared similar, the predicted prevalence of symptomatic allergy levelled off or even decreased at $\mathrm{IgG} 4>$ $300 \mathrm{AU}$ in those reporting no contact with rats.

\section{IgG4 antibody levels and incident rat allergy}

The association between IgG4 antibody levels and newly occurring sensitization and rat allergy was studied in workers who were not sensitized or did not report allergic respiratory symptoms to rat allergens at the first survey.

Cumulative incidence of a positive SPT to rat urine or rat hair in the non-sensitized cohort was 7.9\% (25/315) over a period (mean [SD]) of 1.7 [0.5] years. In simple unadjusted analysis both female gender $(\mathrm{OR}[95 \% \mathrm{CI}]=3.0[1.2-7.8])$ and atopy $(\mathrm{OR}[95 \% \mathrm{CI}]=2.3[0.9-5.9])$ appeared to be risk factors, while smoking was no relevant explanatory factor $(\mathrm{OR}[95 \% \mathrm{CI}]=1.4[0.5-3.5])$

An elevated IgG4 antibody level at baseline was a strong predictor of sensitization during follow-up in atopic workers. Sensitization risk increased from $4 \%(1 / 27)$ in those without detectable IgG4 to $28 \%(9 / 32)$ in those with $\mathrm{IgG} 4>100 \mathrm{AU}$ (highest tertile of detectable $\mathrm{IgG} 4$ ). In non-parametric regression analysis the association between log-transformed IgG4 and sensitization risk appeared to be well described by a linear logistic model (Fig. 5). From this model the OR $[95 \% \mathrm{CI}]$ for a 10 -fold increase in IgG4 titre was calculated as $2.4[1.4-4.2]$ for atopic and 1.0 [0.6-1.7] for non-atopic workers. These estimates did not change when exposure to RUA was included in the model. 


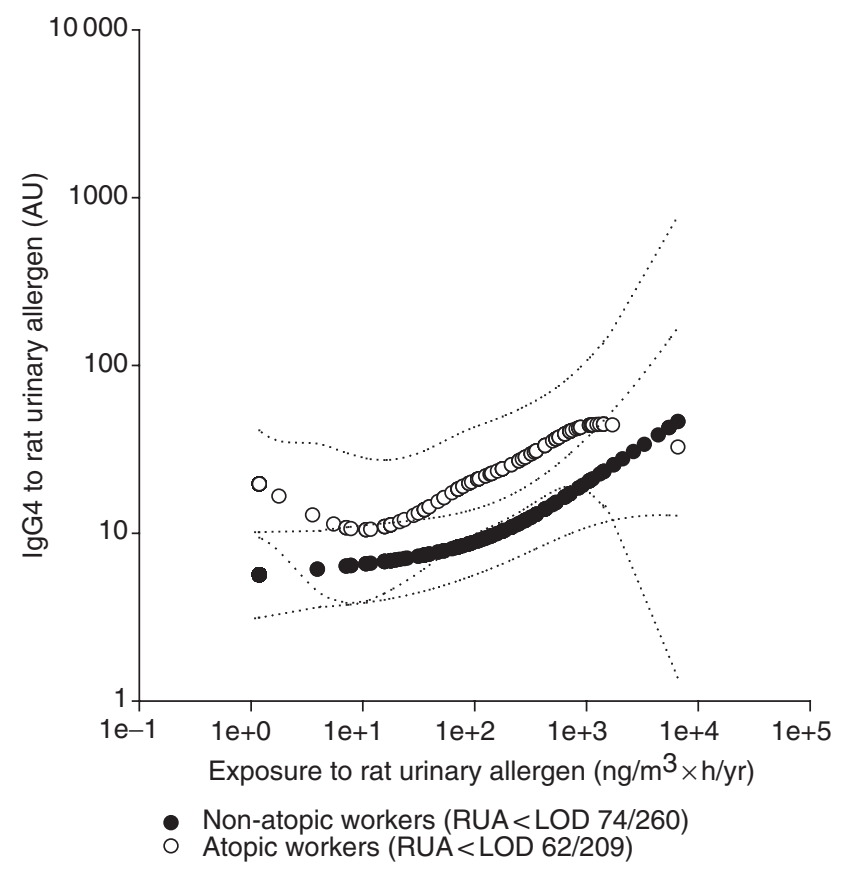

Fig. 2. Smoothed (cubic spline) plots with pointwise \pm 2 SE bands of exposure and IgG4 to rat urinary allergen (RUA) in atopic and non-atopic laboratory animal workers. Smoothness of the plot was determined by generalized cross-validation. Subjects not working with rats during the year preceding the study were assigned an arbitrary exposure of $1.18 \mathrm{ng} /$ $\mathrm{m}^{3} \times \mathrm{h} / \mathrm{yr}$ before log transformation (see Materials and methods section). The number of subjects not working with rats is indicated in the legend as: RUA $<$ LOD number of subjects/total number of subjects in group. For higher exposures a single dot represents one to 14 subjects (median $=2$ ), depending on the number of subjects with identical estimated exposure in a particular group.

Cumulative incidence of symptomatic respiratory rat allergy in the non-symptomatic cohort was $6.8 \%(27 / 399)$ over a period (mean [SD]) of 1.8 [0.4] years. Single most important determinant was a positive SPT to rat allergens at the first survey $(\mathrm{OR}[95 \% \mathrm{CI}]=16.5$ [6.2-44]; SPT results available for 25 cases and 350 non-cases).

The median titre of IgG4 in subjects with detectable levels of IgG4 was $40 \mathrm{AU}$. Risk of becoming symptomatic in ratsensitized subjects was high in subjects with titres of $\mathrm{IgG} 4$ above this level $(10 / 23=43 \%)$, lower in those with detectable IgG4 below this level $(2 / 12=17 \%)$, and highest in subjects with IgG4 below the detection limit $(2 / 4=50 \%)$. Although not statistically significant and based on just a few observations, this non-linear response was also evident when nonparametric regression analysis was used to model the doseresponse relation (Fig. 5) and persisted when subjects with IgG4 below the detection limit were removed from the analysis. Risk of becoming symptomatic was low in those who were not sensitized (approximately 3-4\%), and was not associated with IgG4 level.

\section{Discussion}

The results of this study strongly suggest that IgG4 antibodies to RUA cannot explain the absence of a dose response between exposure to rat allergens and rat allergy in long- term- exposed LA workers. IgG4 antibodies also do not protect against developing respiratory rat allergy. Titres of IgG4 showed a strong and positive dose response with exposure to RUA, and were highest in subjects who were symptomatic and sensitized to rat allergens. Prevalence of a positive SPT or respiratory symptoms to rat allergens did seem to level off or decrease only at the highest IgG4 titres in subjects who did not work with rats. High levels of $\mathrm{IgG} 4$ predicted newly occurring sensitization and development of respiratory symptoms in atopic and sensitized workers during follow-up.

This is the first longitudinal study to look at the relation between high levels of allergen-specific IgG4 and development of allergy and allergic respiratory disease in an open working population. This was a relatively large study and exposure to rat allergens had been characterized extensively. However, many subjects had been working with rats for several years prior to enrolment in the study and healthy worker selection may have caused those who were (more) sensitive to the effects of allergen exposure to leave their job either before or during the study. As those lost to follow-up were more often sensitized to rats and also reported respiratory symptoms more often [16], the incidence of sensitization (and symptomatic allergy) as reported here are probably lower than what they would have been in a cohort of novice LA workers.

As subjects were aware of their SPT status at the start of follow-up and symptoms were not confirmed by objective measurements, those with a positive SPT could have been more likely to attribute any respiratory symptoms to working with rats, thus possibly biasing the relation between exposure to rats and onset of respiratory symptoms. It is unlikely that this could explain the association between titres of $\mathrm{IgG} 4$ and onset of respiratory symptoms in sensitized workers we found, as this relation was still evident when analysed in different strata based on (partly self-reported) exposure levels. Use of an arbitrary cut-point for a positive SPT in defining the population at risk for sensitization could have biased our results, since those with a weak SPT reaction who were nonetheless considered SPT negative at the first survey were more likely to become sensitized during follow-up. However, titres of IgG4 were not associated with weal diameter in those with a negative SPT, and exclusion of workers with a marginal skin reaction from the follow-up cohort or using a more stringent definition for a positive SPT had only minor effects on the strength of the associations we found between IgG4 and rat allergy.

Measurement error may have caused misclassification of exposure and response, but will probably have been nondifferential and therefore cannot explain why we found positive associations between titres of IgG4 and both prevalent and newly occurring rat allergy.

Although we found a strong association between titres of IgG4 and incidence of sensitization or symptomatic rat allergy, we do not consider IgG4 to be a strong causal factor as these associations were limited to those with a positive SPT to common and/or rat allergens at the first survey. As reported in other studies [17], presence of IgG4 in subjects who were not sensitized did not increase the risk of becoming symptomatic when working with rats. Several authors have suggested that the presence of high levels of specific IgG4 might be a marker for prolonged and/or high level exposure 


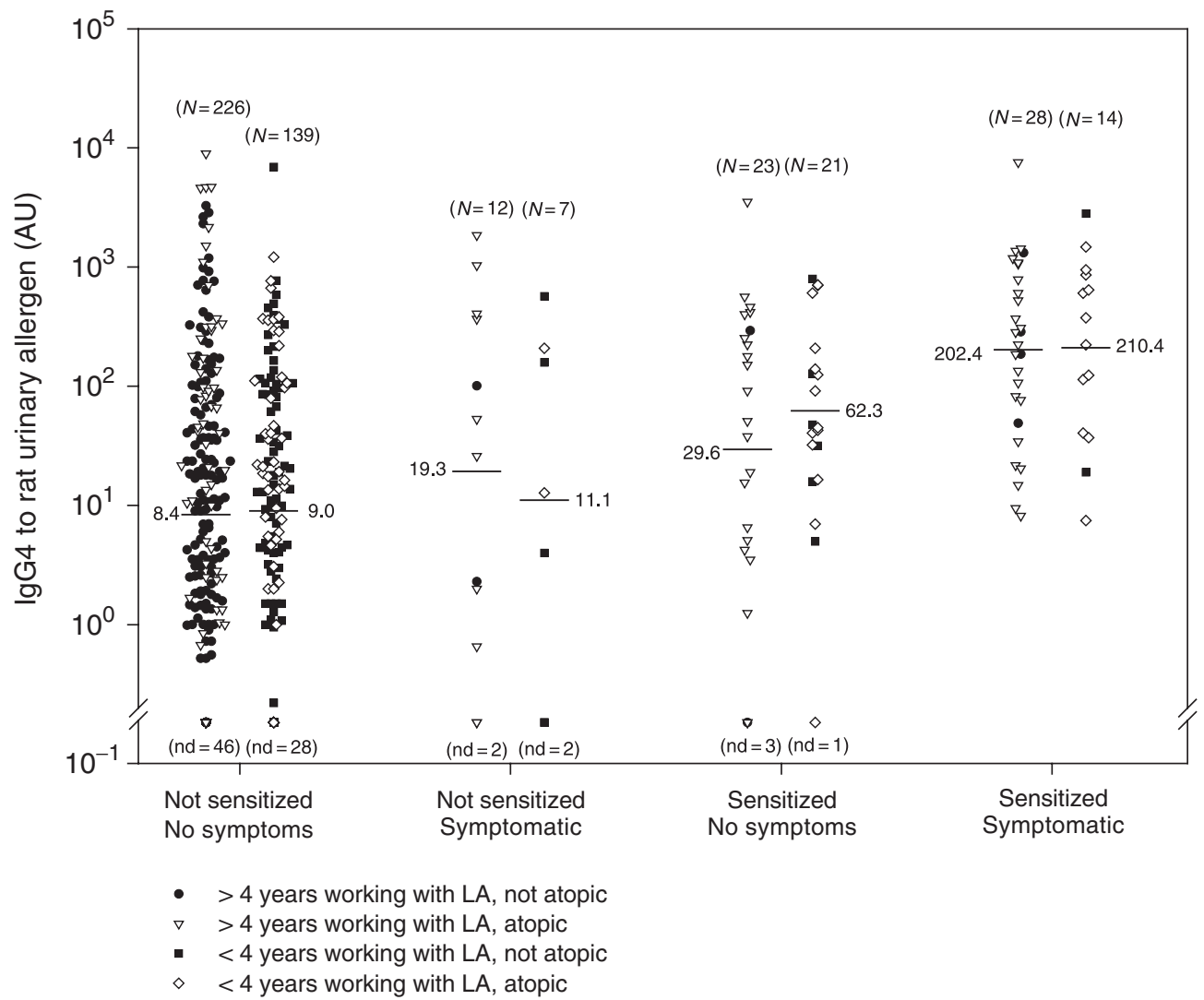

Fig. 3. IgG4 to rat urinary allergen in sensitized and non-sensitized laboratory animal (LA) workers with and without respiratory symptoms. $N$, number of subjects in group; nd, number of subjects with IgG4 below the detection limit.
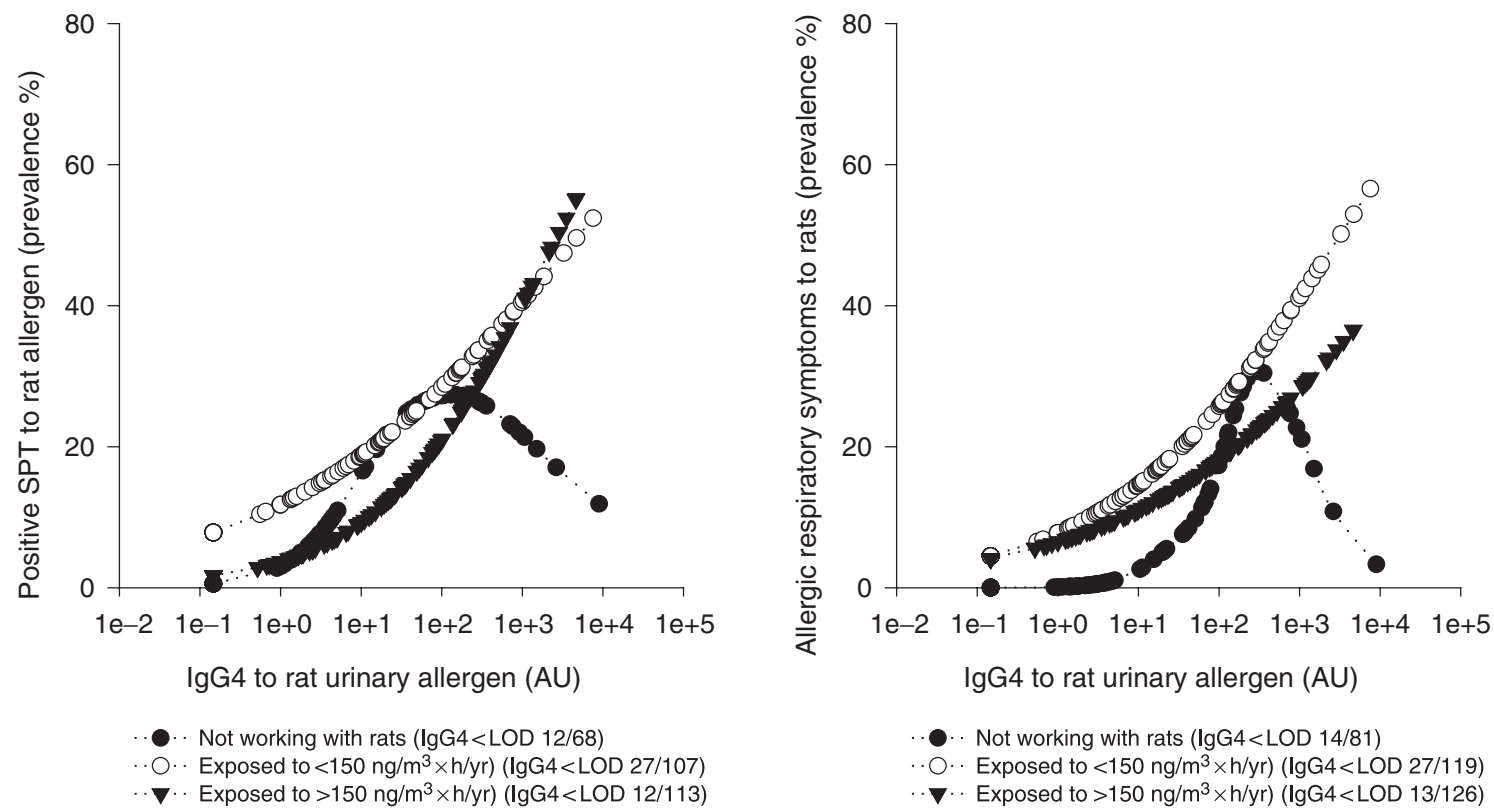

Fig. 4. Smoothed dose-response curves for IgG4 to rat urinary allergen (RUA) and prevalent sensitization and symptomatic rat allergy at different levels of exposure to RUA. Smoothness of the plot determined by generalized cross-validation except where indicated with a (see Materials and methods section). Subjects with IgG4 below the limit of detection were given an arbitrary titre of $0.001 \mathrm{AU}$ before log transformation (see Materials and methods section). The number of subjects with IgG4 below the detection limit is indicated in the legend as: IgG4<LOD number of subjects/total number of subjects in group. For IgG4 titres above the detection limit a single dot represents one to four subjects $(90 \%$ quantile $=1)$, depending on the number of subjects with identical IgG4 titre in a particular group.

to the antigen involved $[7-9,18,19]$. The fact that we found a strong association between IgG4 and sensitization or symptomatic allergy even within different RUA exposure strata suggests that there must be additional factors to explain this association. Higher levels of antigen-specific IgG4 in atopic or allergic subjects when compared with non-atopic or 

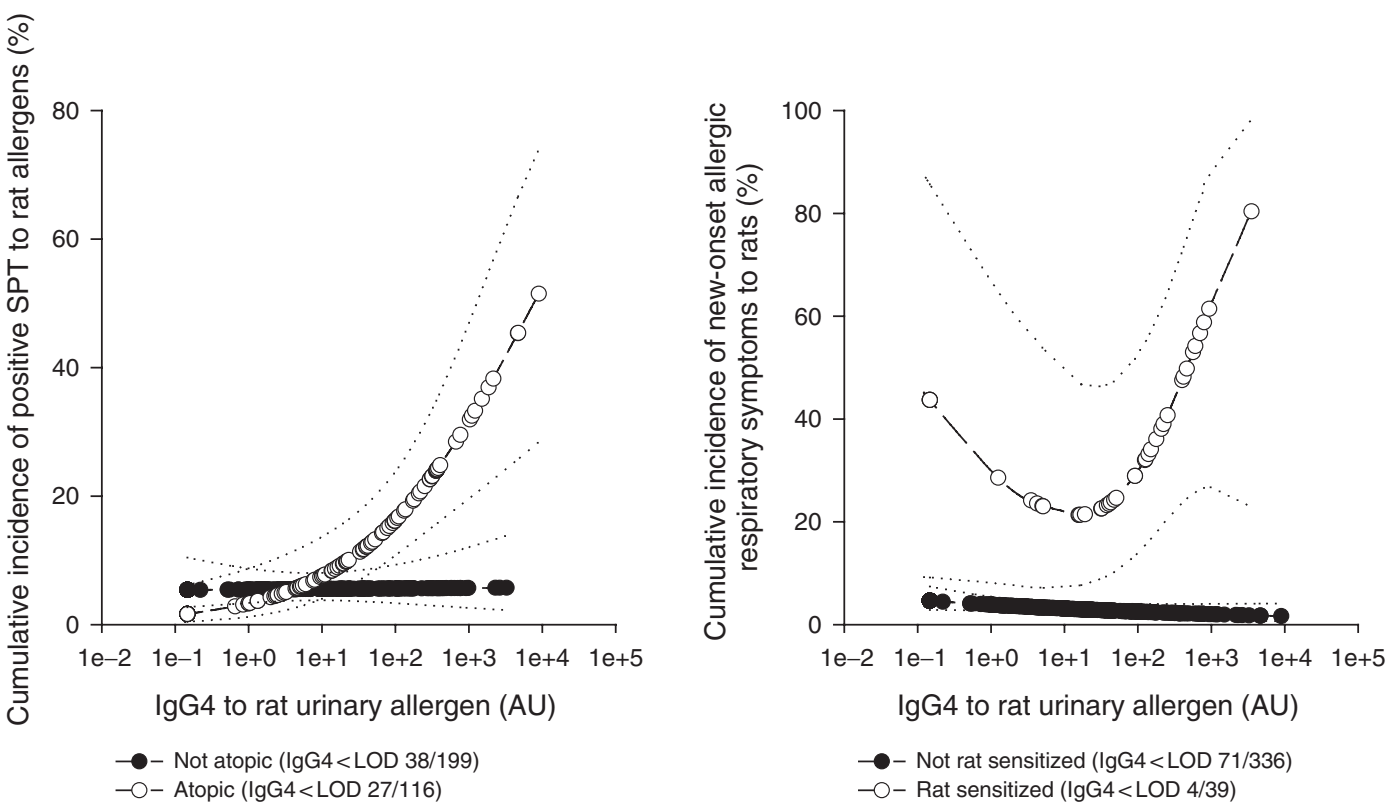

Fig. 5. Smoothed dose-response curves with pointwise \pm 2 SE bands for IgG4 to rat urinary allergen and newly occurring sensitization and symptomatic allergy to rats. Smoothness of the plot determined by generalized cross-validation. Subjects with IgG4 below the limit of detection were given an arbitrary titre of $0.001 \mathrm{AU}$ before log transformation (see Materials and methods section). The number of subjects with IgG4 below the detection limit is indicated in the legend as: IgG4<LOD number of subjects/total number of subjects in group. For IgG4 titres above the detection limit a single dot represents one to seven subjects $(90 \%$ quantile $=1)$, depending on the number of subjects with identical IgG4 titre in a particular group.

non-allergic subjects have also been reported by others [2023]. As both IgG4 and IgE switching are stimulated by IL-4 [24] we believe that IgG4 should be regarded as a marker that combines aspects of exposure and susceptibility. This is also suggested by the fact that the dose response between exposure to RUA and titres of IgG4 was similar for non-atopic and atopic subjects, but at different response levels.

Our finding of a strong positive relation between $\mathrm{IgG} 4$ and sensitization and symptoms of allergy to rats is at variance with the results for $\operatorname{IgG}(4)$ to cat allergen and sensitization and asthma in the study by Platts-Mills et al. [5]. In their study, $\operatorname{IgG}(4)$ to the major cat allergen Fel d 1 was highest in children in the highest exposure category, but was associated with a lower prevalence of $\operatorname{IgE}$ sensitization to Fel d 1 and a low risk of asthma. In contrast, $\operatorname{IgG}(4)$ to the group 1 mite allergens was positively associated with both exposure and prevalence of sensitization to mite. They suggested that the much higher exposure to cat allergens and lack of significant enzymatic activity of the major cat allergen Fel d 1 might explain these different relations [25]. This hypothesis is not supported by our results as exposure to rat allergens was at least comparable to if not higher than that for cat allergen and the major RUAs do not appear to possess significant enzymatic activity [26]. The fact that high titres of IgG4 predicted newly occurring positive SPT reactions against rat allergens also argues against $\mathrm{IgG} 4$ being the result of a 'modified' Th2 response, as strong IgG4 responses in these workers were present before IgE responses became evident.

There are many differences between this study and that by Platts-Mills et al. [5] that could explain our different results. Subjects in our study were older, were probably exposed more intermittently, and exposed to a variety of other chemicals. It is also possible that our use of a crude urine extract instead of purified allergens may have affected our results. It has been suggested that epitope specificity for IgE and IgG4 may be largely different [14] and it is therefore possible that the use of purified allergens would have shown a protective effect of IgG4 antibodies raised against the major allergenic epitopes on prevalent or newly occurring sensitization.

In a recent study by Doekes et al. [27] high levels of IgG4 to pig urinary protein were associated with lower prevalences of respiratory symptoms and bronchial hyper-responsiveness in two different populations of pig farmers. However, respiratory symptoms in farmers are often non-allergic and specific sensitization to work-related allergens is rare. Assuming that IgG4 titres are associated with exposure in this population, it is difficult to see how a blocking effect of IgG4 antibodies would have a major impact on the presence of primarily nonallergic symptoms. It could therefore be that high levels of IgG4 in this population reflect (relatively) low exposure to agents with a Th1 skewing effect (like endotoxin) that might have more impact on respiratory health for people in this working environment.

Our results are in agreement with the view that an $\mathrm{IgG} 4$ response should not be regarded as harmful per se, as high IgG4 titres were not associated with adverse effects in subjects who were not atopic or were not sensitized to rat allergens.

\section{Acknowledgements}

This work was supported by Grant No. 99.33 from the Dutch Asthma Foundation.

\section{References}

1 Baur X, Chen Z, Liebers V. Exposure-response relationships of occupational inhalative allergens. Clin Exp Allergy 1998; 28:537-44. 
2 Custovic A, Murray CS. The effect of allergen exposure in early childhood on the development of atopy. Curr Allergy Asthma Rep 2002; 2:417-23.

3 Heederik D, Thorne PS, Doekes G. Health-based occupational exposure limits for high molecular weight sensitizers: how long is the road we must travel? Ann Occup Hyg 2002; 46:439-46.

4 Heederik D, Houba R. An exploratory quantitative risk assessment for high molecular weight sensitizers: wheat flour. Ann Occup Hyg 2001; 45:175-85.

5 Platts-Mills T, Vaughan J, Squillace S, Woodfolk J, Sporik R. Sensitisation, asthma, and a modified Th2 response in children exposed to cat allergen: a population-based cross-sectional study. Lancet 2001; 357:752-6.

6 Anyo G, Brunekreef B, de Meer G, Aarts F, Janssen NA, van Vliet P. Early, current and past pet ownership: associations with sensitization, bronchial responsiveness and allergic symptoms in school children. Clin Exp Allergy 2002; 32:361-6.

7 Tiikkainen U, Klockars M. Clinical significance of IgG subclass antibodies to wheat flour antigens in bakers. Allergy 1990; 45:497-504

8 Zock JP, Doekes G, Heederik D, Van Zuylen M, Wielaard P. Airborne dust antigen exposure and specific $\mathrm{IgG}$ response in the potato processing industry. Clin Exp Allergy 1996; 26:542-8.

9 Yokota K, Yamaguchi K, Takeshita T, Morimoto K. The significance of specific IgG4 antibodies to methyltetrahydrophthalic anhydride in occupationally exposed subjects. Clin Exp Allergy 1998; 28:694-701.

10 Frew AJ. 25 Immunotherapy of allergic disease. J Allergy Clin Immunol 2003; 111:S712-9.

11 Hollander A, Heederik D, Doekes G. Respiratory allergy to rats: exposure-response relationships in laboratory animal workers. Am J Respir Crit Care Med 1997; 155:562-7.

12 Hollander A, Doekes G, Heederik D. Cat and dog allergy and total $\mathrm{IgE}$ as risk factors of laboratory animal allergy. J Allergy Clin Immunol 1996; 98:545-54.

13 Hollander A, Van Run P, Spithoven J, Heederik D, Doekes G. Exposure of laboratory animal workers to airborne rat and mouse urinary allergens. Clin Exp Allergy 1997; 27:617-26.

14 Kobayashi I, Sakiyama Y, Tame A, Kobayashi K, Matsumoto S. $\mathrm{IgE}$ and IgG4 antibodies from patients with mite allergy recognize different epitopes of Dermatophagoides pteronyssinus group II antigen (Der p2). J Allergy Clin Immunol 1996; 97:638-45.
15 El Shami AS, Alaba O. Liquid-phase in vitro allergen-specific IgE assay with in situ immobilization. Adv Biosci 1989; 74:191-201.

16 Portengen L, Hollander A, Doekes G, de Meer G, Heederik D. Lung function decline in laboratory animal workers: the role of sensitisation and exposure. Occup Environ Med 2003; 60:870-5.

17 Platts-Mills TA, Longbottom J, Edwards J, Cockroft A, Wilkins S. Occupational asthma and rhinitis related to laboratory rats: serum IgG and IgE antibodies to the rat urinary allergen. J Allergy Clin Immunol 1987; 79:505-15.

18 Aalberse RC, van der Gaag R, van Leeuwen J. Serologic aspects of IgG4 antibodies. I. Prolonged immunization results in an IgG4restricted response. J Immunol 1983; 130:722-6.

19 Van der Zee JS, Aalberse RC. The role of IgG in immediate-type hypersensitivity. Eur Respir J 1991; 13 (Suppl.):91s-6s.

20 Botham JW, McCall JC, Teasdale EL, Botham PA. The relationship between exposure to rats and antibody production in man: IgG antibody levels to rat urinary protein. Clin Exp Allergy 1989; 19:437-41.

21 Kemeny DM, Urbanek R, Ewan P. et al. The subclass of IgG antibody in allergic disease: II. The IgG subclass of antibodies produced following natural exposure to dust mite and grass pollen in atopic and non-atopic individuals. Clin Exp Allergy 1989; 19:545-9.

22 Tame A, Sakiyama Y, Kobayashi I, Terai I, Kobayashi K. Differences in titres of $\mathrm{IgE}, \mathrm{IgG} 4$ and other IgG subclass anti-Der $\mathrm{p}$ 2 antibodies in allergic and non-allergic patients measured with recombinant allergen. Clin Exp Allergy 1996; 26:43-9.

23 Bottcher MF, Jenmalm MC, Bjorksten B. Immune responses to birch in young children during their first 7 years of life. Clin Exp Allergy 2002; 32:1690-8.

24 Vercelli D. One cytokine, two isotypes: a trojan horse, pandora's box, and an evolving paradigm. Am J Respir Crit Care Med 2000; 162:S86-90.

25 Platts-Mills TA, Perzanowski M, Woodfolk JA, Lundback B. Relevance of early or current pet ownership to the prevalence of allergic disease. Clin Exp Allergy 2002; 32:335-8.

26 Wood RA. Laboratory animal allergens. ILAR J 2001; 42:12-6.

27 Doekes G, Preller L, Snippe R, Iversen M, Vogelenzang P, Heederik D. IgG4 responses to work-related aeroallergens: protection against allergic and inflammatory airway disease. J Allergy Clin Immunol 2002; 109:S90. 